\title{
First-Person Vision Based Prediction of Information Seeking and Use Behavior in Library
}

\author{
Kazushi OKAMOTO*1 • Kazuhiko KAWAMOTO*2
}

\begin{abstract}
We propose an automated method for predicting subject behaviors based on first-person vision in an area surrounding bookshelves. The proposal classifies each frame within a movie recorded using a head-mounted camera to the six primitive behaviors according to naive Bayes nearest-neighbor method (NBNN). A prediction experiment is conducted using two image sequences recorded by a head-mounted camera. The experimental results confirm that the average classification rates for NBNN with random sampling (including principal components analysis) are improved from 0.09 to 0.13 for one data set and from 0.03 to 0.08 for the other data set compared with the bag-of-features and support vector machine combination results.
\end{abstract}

Keywords: classification, information seeking and use behavior, library, naive Bayes nearest-neighbor, principal components analysis

\section{Introduction}

The survey of user behavior when searching for specific topics/themes in libraries is a research field in library and information science. The purposes of such surveys are to analyze how users use a library and acquire information and to improve the quality of library services according to the results obtained. In the current survey style, investigators observe subjects or users by filling out survey sheets or recording through handy-camera or headmounted camera [1-3]; thereby, enabling automatic recording and detection of users' behaviors help survey planning in terms of the immensity and efficiency.

We propose an automated method for detecting behaviors from recorded movies in which subjects wear a head-mounted camera. Series of detected behaviors is useful for example at interview planning and evidences to confirm questionnaire results. This method predicts behavior in an input frame by using the naive Bayes nearest-neighbor method (NBNN) [4]. The purpose of this study is to develop a behavior prediction method with high prediction accuracy and easy implementation for investigators. We define six primitive behaviors as the target user behaviors in an area surrounding bookshelves: aisle, walk, slant, face, pickup, and browse. A prediction experiment is conducted using two image sequences recorded through a head-mounted camera. The image sequences are recorded under the condition in which a subject freely searches for books in an area surrounding bookshelves without objectives. Further, the classification accuracy of the proposal is measured in the experiment by using the prepared training and testing examples from the image sequences. In addition, a baseline method based on bag-of-features (BoF) [5] and support vector machine (SVM) [6] and different versions of the proposal,

\footnotetext{
*1 Department of Informatics, Graduate School of Informatics and Engineering, The University of Electro-Communications

*2 Institute of Management and Information Technologies, Chiba University
}

in which random sampling or principal components analyses are applied, are validated for classification accuracy.

The remainder of this paper is organized as follows: Section 2 defines the six primitive behaviors; Section 3 describes the classification model of this research; Section 4 shows the experimental conditions; and Section 5 analyzes classification accuracy for the proposal and the baseline method.

\section{Definition of Primitive Behaviors}

In libraries, bookshelves are placed beside each other, and blind areas exist, which may prove a hindrance in sequentially recording subjects through fixed cameras. It is possible for a researcher to track a subject with a handy-camera; however, this approach is difficult in terms of long-time tracking and increase in the number of subjects.

We assume that a subject wears a head-mounted camera, which records sequential behaviors in first-person vision. The advantages of this assumption are as follows: (1) subjects are comfortably able to use library without being aware of being watched by the researcher; (2) recording is not affected by blind areas; (3) head-mounted cameras can be prepared at low cost; (4) increase in the number of subjects can be addressed by increase in the number of cameras.

Recorded movies are used for automated prediction of subjects' behaviors based on a classification model. Moreover, studies of information seeking and use behavior in libraries cover all processes to obtain the information; however, this study focuses only on six types of primitive behaviors of subjects in an area surrounding bookshelves: aisle - move in an aisle; walk - move between bookshelves; slant - turn head toward bookshelves; face - search books; pickup - pick up or put back a book; browse - read a book. Example frames for each primitive behavior are shown in Fig. 1.

\section{Classification Model}

Each frame within a movie recorded by a head-mounted cam- 


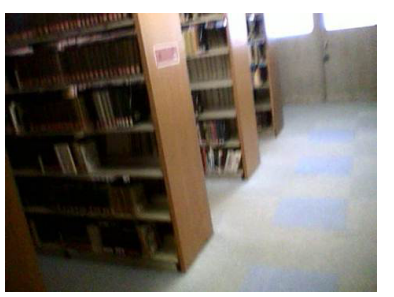

aisle

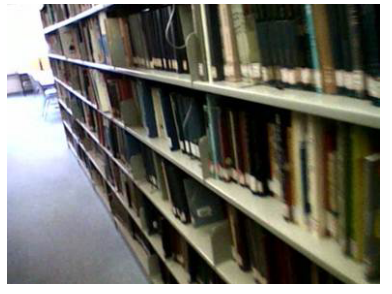

slant

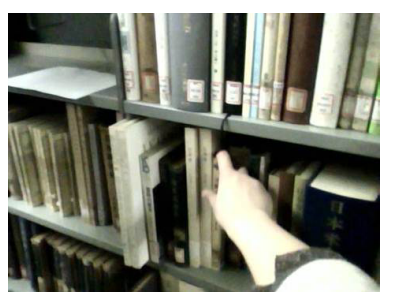

pickup

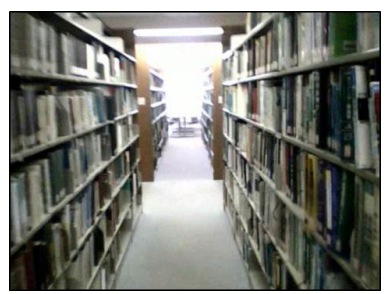

walk

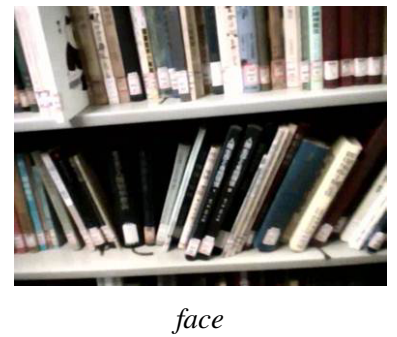

face

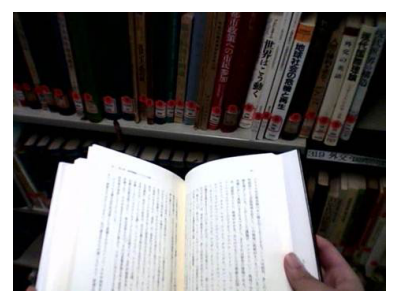

browse
Fig. 1 Example frames of the six primitive behaviors.

era is classified into a primitive behavior based on supervised learning. The training examples for learning consist of pairs of frames and labels obtained by labeling each frame within the movies recorded in advance. A set of training examples $T$ is defined using a set of ordered frames $X \subset \Omega(\Omega$ : a set of frames) and a set of labels $Y=\{$ aisle, walk, slant, face, pickup, browse $\}$ :

$$
T=\left\{\left(x_{1}, y_{1}\right),\left(x_{2}, y_{2}\right), \ldots,\left(x_{i}, y_{i}\right), \ldots,\left(x_{t}, y_{t}\right)\right\}
$$

where $x_{i} \in X$ and $y_{i} \in Y$. The classification model in this study is formalized as

$$
\hat{y}=\underset{y \in Y}{\operatorname{argmax}} f(T, q ; \boldsymbol{\theta}),
$$

with an unlabeled input (query) frame $q$, a predicted label $\hat{y}$, hyper-parameters $\boldsymbol{\theta}$, and a scoring function $f$.

We consider that the classification model is simple with few hyper-parameters as possible, that is, reduced user decisions. The conditions are effective for reducing survey costs for librarians who plan and conduct this type of survey because they are not always familiar with machine learning technology, and parameter selection requires professional knowledge and experience. First, we assume to ignore relationships between pre- and post-frames for simplification, while considering the importance of utilizing time series. We then propose a classification model based on the NBNN [4]. NBNN is used because it does not require hyper-parameters for classification and it is expected to contribute high-level classification accuracy according to previous studies. NBNN is formalized as
Table 1 Numbers of frames in the training and testing data sets.

\begin{tabular}{l|cc}
\multicolumn{3}{c}{ (1) Training examples } \\
\hline & $\begin{array}{c}\text { Data } \\
\text { set A }\end{array}$ & $\begin{array}{c}\text { Data } \\
\text { set B }\end{array}$ \\
\hline aisle & 65 & 76 \\
walk & 54 & 67 \\
slant & 62 & 61 \\
face & 56 & 63 \\
pickup & 52 & 100 \\
browse & 82 & 100 \\
\hline
\end{tabular}

\begin{tabular}{l|cc}
\multicolumn{3}{c}{ (2) Testing examples } \\
\hline & $\begin{array}{c}\text { Data } \\
\text { set A }\end{array}$ & $\begin{array}{c}\text { Data } \\
\text { set B }\end{array}$ \\
\hline aisle & 29 & 42 \\
walk & 18 & 40 \\
slant & 17 & 49 \\
face & 35 & 63 \\
pickup & 37 & 59 \\
browse & 24 & 58 \\
\hline
\end{tabular}

$$
\hat{y}=\underset{y \in Y}{\operatorname{argmin}} \sum_{j=1}^{n}\left\|d_{j}-\mathrm{NN}_{y}\left(d_{j}\right)\right\|^{2},
$$

where $n$ is the number of local features in a query frame $q$ ( $\left.=\left\{d_{1}, d_{2}, \ldots, d_{n}\right\}\right), d_{j}$ is the $j$-th local feature in $q,\|\cdot\|$ is the Euclidean norm, and $\mathrm{NN}_{y}$ is the nearest local feature with label $y$ on the Euclidean space. Local features are generally calculated using the scale-invariant feature transform [7] or speeded up robust features (SURF) [8]. From the definition, NBNN classifies the query only depending on distances among local features. In addition, NBNN does not require quantization process compared with BoF model [5], and it is expected to eliminate adverse effects for classification accuracy caused by quantization error.

\section{Classification Experiment}

\subsection{Training and Testing Examples}

As a simulation of information seeking and use behavior in library, a subject freely searches books in an area surrounding bookshelves without objectives, and the series of behaviors are recorded using a head-mounted camera in first-person vision. Two movies were obtained at the Chiba University Library on December 2011 and February 2013, and we prepare two data sets: one, called Data set A, is constructed from the movie taken in December 2011; one, called Data set B, is constructed from the movie taken in February 2013. Each movie consists of a part for training and a part for testing, respectively, and each part contains recorded subject's behaviors. Then, frames within the part are treated as training or testing examples. Each frame within a movie is labeled manually with one of the six classes. The Table 1 shows the numbers of labeled frames within the two data sets. The camera used is over the ear type: a Looxcie product. It outputs a $640 \times 480$ sized color movie with $30 \mathrm{fps}$, and the obtained movie is down sampled to $3 \mathrm{fps}$.

\subsection{Experimental Condition}

In this study, SURF implemented in OpenCV (C++ version) is used for local feature description, and the number of local feature dimensions is 128 . The constructor of SURF class is called with the following parameters: hessianThreshold $=500$; nOctaves $=4$; nOctaveLayers $=2$; extended $=$ false. The local features are uniformly extracted from a frame through grid sampling per 10 pixels, i.e., the number of local features per frame is 3,072.

According to Table 1, the total number of local features for each class is $159,744(=52 \times 3,072)$ to $307,200(=100 \times 3,072)$. While using the naive Bayes classifier, it is generally recom- 
mended to equalize the numbers of local features among each class. Further, application of the principal components analysis (PCA) is expected to reduce the computational cost for classification. Therefore, we validate the classification accuracy by randomly sampling either 50,000, 100,000, or 150,000 features per class and applying PCA. In this experiment, PCA reduces the number of local feature dimensions from 128 to 31 for Data set $\mathrm{A}$ and 32 for Data set B, and their cumulative contribution ratios are more than $90 \%$.

As a baseline, we also validate the classification accuracy in which a classification model consists of BoF [5] and SVM [6]. Several applications recognize BoF and SVM in the object category and considerable knowledge exists. BoF describes each frame within training examples as a histogram of included visual words, which are a type of quantized vectors. They correspond to representative vectors for a set of local features from an entire collection. In usage of BoF, SURF is also used to obtain local features with same parameters of the NBNN's case. When using $\mathrm{BoF}$, we have previously checked classification accuracy for 500, 1,000, and 3,000 visual words cases by using Data set A. According to the result, the case has best classification accuracy; therefore, we select 3,000 as the number of visual words. The $k$-means++ [9] is used for vector quantization. The kernel function used in SVM is a spatial pyramid match kernel [10] based on histogram intersection [11].

\section{Analysis of Classification Accuracy}

The confusion matrices for the combination of BoF and SVM for Data sets A and B are shown in Table 2. The Table 3 summarizes average classification rates and standard deviations for each data set and each NBNN versions. The eight versions of NBNN in Table 3: original (no PCA and random sampling), PCA only (applied PCA), RS (applied random sampling, sampling size is either 50,000, 100,000, or 150,000), and PCA-RS (applied PCA and RS). In addition, Tables 4 and 5 show two confusion matrices which achieve the best average classification rates in terms of RS and PCA-RS. In Tables 2, 4, and 5, the symbols (a), (w), (s), (f), (p), and (b) correspond to aisle, walk, slant, face, pickup, and browse, respectively.

Form Tables 3, 4, and 5, we suggest the following:

- the average classification rates for NBNN with random sampling (including the PCA version) are improved from 0.09 to 0.13 for Data set A and from 0.03 to 0.08 for Data set B compared with BoF and SVM results

- random sampling with or without PCA improves the classification rates compared with the original method and PCA only versions

- the number of local features in each class should be more or less the same

- application of PCA or change in sampling size almost do not affect the classification rates.

Since random sampling works improvement of classification accuracy, random sampling is necessary for using NBNN, and we
Table 2 Confusion matrices for classification rates on Data sets $\mathrm{A}$ and $\mathrm{B}$ with BoF and SVM.

\begin{tabular}{c|cccccc}
\multicolumn{7}{c}{ Data set A } \\
\hline & (a) & (w) & (s) & (f) & (p) & (b) \\
\hline (a) & $\mathbf{0 . 4 8}$ & 0.38 & 0.07 & - & 0.07 & - \\
(w) & 0.22 & $\mathbf{0 . 0 6}$ & 0.56 & 0.11 & 0.06 & - \\
(s) & 0.35 & 0.06 & $\mathbf{0 . 2 9}$ & 0.24 & 0.06 & - \\
(f) & - & - & - & $\mathbf{0 . 9 1}$ & 0.09 & - \\
(p) & - & 0.03 & - & 0.49 & $\mathbf{0 . 4 6}$ & 0.03 \\
(b) & - & - & - & 0.04 & 0.08 & $\mathbf{0 . 8 8}$ \\
\hline
\end{tabular}

avg. classification rate: 0.51 , std. dev.: 0.33

\begin{tabular}{c|cccccc}
\multicolumn{7}{c}{ Data set B } \\
\hline & (a) & (w) & (s) & (f) & (p) & (b) \\
\hline (a) & $\mathbf{0 . 7 9}$ & 0.05 & 0.12 & 0.05 & - & - \\
(w) & - & $\mathbf{0 . 6 5}$ & 0.35 & - & - & - \\
(s) & 0.02 & 0.20 & $\mathbf{0 . 6 5}$ & 0.12 & - & - \\
(f) & - & - & - & $\mathbf{0 . 9 8}$ & 0.02 & - \\
(p) & - & - & 0.02 & 0.70 & $\mathbf{0 . 2 7}$ & 0.02 \\
(b) & - & - & 0.02 & 0.09 & 0.05 & $\mathbf{0 . 8 5}$ \\
\hline avg. classification rate: 0.70, std. dev.: 0.24
\end{tabular}

Table 3 Average classification rates for Data sets A and B with the eight NBNN versions.

\begin{tabular}{l|ll}
\hline & Data set A & Data set B \\
\hline Original & $0.47 \pm 0.43$ & $0.54 \pm 0.44$ \\
PCA only & $0.47 \pm 0.44$ & $0.50 \pm 0.44$ \\
RS $(50,000)$ & $0.59 \pm 0.30$ & $0.75 \pm 0.19$ \\
RS $(100,000)$ & $0.61 \pm 0.30$ & $0.77 \pm 0.19$ \\
RS $(150,000)$ & $0.62 \pm 0.29$ & $0.78 \pm 0.21$ \\
PCA-RS $(50,000)$ & $0.63 \pm 0.27$ & $0.73 \pm 0.19$ \\
PCA-RS $(100,000)$ & $0.64 \pm 0.27$ & $0.74 \pm 0.19$ \\
PCA-RS $(150,000)$ & $0.60 \pm 0.30$ & $0.77 \pm 0.18$ \\
\hline
\end{tabular}

Table 4 Confusion matrices for classification rates on Data set A with the two NBNN versions.

\begin{tabular}{c|cccccc}
\multicolumn{7}{|c}{ RS $(150,000)$} \\
\hline & (a) & (w) & (s) & (f) & (p) & (b) \\
\hline (a) & $\mathbf{1 . 0}$ & - & - & - & - & - \\
(w) & 0.11 & $\mathbf{0 . 3 3}$ & 0.56 & - & - & - \\
(s) & 0.41 & 0.06 & $\mathbf{0 . 5 3}$ & - & - & - \\
(f) & 0.06 & - & 0.26 & $\mathbf{0 . 4 6}$ & 0.20 & 0.03 \\
(p) & - & - & 0.03 & 0.32 & $\mathbf{0 . 4 3}$ & 0.22 \\
(b) & - & - & - & 0.04 & - & $\mathbf{0 . 9 6}$ \\
\hline
\end{tabular}

\begin{tabular}{c|cccccc}
\multicolumn{7}{c}{ PCA-RS $(100,000)$} \\
\hline & (a) & (w) & (s) & (f) & (p) & (b) \\
\hline (a) & $\mathbf{1 . 0}$ & - & - & - & - & - \\
(w) & 0.11 & $\mathbf{0 . 3 9}$ & 0.50 & - & - & - \\
(s) & 0.35 & 0.12 & $\mathbf{0 . 5 3}$ & - & - & - \\
(f) & 0.09 & - & 0.14 & $\mathbf{0 . 4 6}$ & 0.29 & 0.03 \\
(p) & - & - & 0.03 & 0.32 & $\mathbf{0 . 5 1}$ & 0.14 \\
(b) & - & - & - & - & 0.04 & $\mathbf{0 . 9 6}$ \\
\hline
\end{tabular}

consider that the improvement is caused by removing effects of overfitting via random sampling. As one of the reasons, we consider that it is effective to make the number of local features within training data of each class of random sampling equal. In addition, random sampling and PCA are effective in reducing computa- 
Table 5 Confusion matrices for classification rates on Data set $B$ with the two NBNN versions.

\begin{tabular}{c|cccccc}
\multicolumn{7}{c}{ RS $(150,000)$} \\
\hline & (a) & (w) & (s) & (f) & (p) & (b) \\
\hline (a) & $\mathbf{0 . 9 3}$ & 0.05 & 0.02 & - & - & - \\
(w) & - & $\mathbf{0 . 8 3}$ & 0.18 & - & - & - \\
(s) & - & 0.43 & $\mathbf{0 . 5 3}$ & 0.04 & - & - \\
(f) & - & 0.02 & - & $\mathbf{0 . 9 5}$ & 0.03 & - \\
(p) & - & - & 0.02 & 0.46 & $\mathbf{0 . 4 9}$ & 0.03 \\
(b) & - & - & - & 0.02 & 0.05 & $\mathbf{0 . 9 3}$ \\
\hline
\end{tabular}

\begin{tabular}{c|cccccc}
\multicolumn{8}{c}{ PCA-RS $(150,000)$} \\
\hline & (a) & (w) & (s) & (f) & (p) & (b) \\
\hline (a) & $\mathbf{0 . 9 0}$ & 0.07 & 0.02 & - & - & - \\
(w) & - & $\mathbf{0 . 7 8}$ & 0.23 & - & - & - \\
(s) & - & 0.33 & $\mathbf{0 . 6 1}$ & 0.06 & - & - \\
(f) & - & 0.02 & 0.02 & $\mathbf{0 . 9 7}$ & - & - \\
(p) & - & - & 0.02 & 0.47 & $\mathbf{0 . 4 9}$ & 0.02 \\
(b) & - & 0.03 & - & 0.02 & 0.10 & $\mathbf{0 . 8 4}$ \\
\hline
\end{tabular}

tional costs for classification because the distance calculations in the classification process are limited and the number of local feature dimensions is reduced.

In these task, application of deep learning approaches such as convolutional neural network is expect to achieve high-level classification accuracy. We, however, think that NBNN should be primarily applied as a classification approach, since usage of NBNN is effective in terms of classification accuracy and without hyper-parameters to be determined.

Classes with the worst classification rates are walk for Data set A and pickup for Data set B, regardless of the classification models BoF and NBNN with random sampling (including the PCA versions). The two actions, that is, information seeking and use behavior in a library, are related to subject's motions; therefore, we consider the use of different types of sensors, such as gyroscope, accelerometer, and magnetic field sensor, to improve classification accuracy for the two actions.

\section{Conclusion}

We propose an automated method for predicting subject behaviors based on first-person vision in an area surrounding bookshelves. The proposal classifies each frame within a movie recorded using a head-mounted camera to the six primitive behaviors according to NBNN [4]. A prediction experiment is conducted using two image sequences recorded by a head-mounted camera under the condition that a subject freely searches for books in an area surrounding bookshelves without objectives.

We measure the classification accuracy of the proposal and a baseline method based on BoF [5] and SVM [6] by prepared training and testing examples from the image sequences. In the proposal, we also evaluate different versions: applied random sampling or PCA. The experimental results confirm that the average classification rates for NBNN with random sampling (including PCA) are improved from 0.09 to 0.13 for Data set A and from 0.03 to 0.08 for Data set B compared with the baseline method. In addition, it is determined that applying PCA and changing the sampling size do not affect almost all classification rates; therefore, random sampling is necessary when using NBNN for obtaining classification accuracy.

In this study, we determine that the classification of walk and pickup class frames is difficult regardless of the classification models and depending on the data sets. The two actions, that is, information seeking and use behavior in a library, are related to a subject's motions; hence, as in the future, we will focus on the usage of different types of sensors such as gyroscope, accelerometer, and magnetic field sensor to improve the classification accuracy for the two actions.

\section{Acknowledgment}

We would like to express our sincere gratitude to Ms. Mayu Horiuchi for contribution to data collection. We would also like to offer our special thanks to Library/Academic Link Center, Chiba University to provide the opportunity to collect data. This work was supported by JSPS KAKENHI Grant Numbers JP23700189, JP25330186, and JP16K00231.

\section{References}

[1] H. Xie: Patterns between interactive intentions and informationseeking strategies, Information Processing \& Management, 38(1), $55-77,2002$.

[2] C. Matsuda: Browsing behavior in information seeking process: On the basis of observation of information-seeking behavior in libraries and bookstores, Library and Information Science, 49, 1-31, 2003 (in Japanese).

[3] H. Terai: A study of information search process in a university library: How do people make use of some qualitatively different information resources?, Annals of Nagoya University Library Studies, 5, 69-82, 2007 (in Japanese).

[4] O. Boiman, E. Shechtman, and M. Irani: In defense of nearestneighbor based image classification, Proceedings of IEEE Conference on Computer Vision and Pattern Recognition, 1-8, 2008.

[5] J. Sivic and A. Zisserman: Video Google: Efficient visual search of videos, Lecture Notes in Computer Science, 4170, 127-144, 2006.

[6] C. C. Chang and C. J. Lin: LIBSVM: A library for support vector machines, ACM Transactions on Intelligent Systems and Technology, 2(3), 1-27, 2011.

[7] D. G. Lowe: Distinctive image features from scale-invariant keypoints, International Journal of Computer Vision, 60(2), 91-110, 2004.

[8] H. Bay, A. Ess, T. Tuytelaars, and L. V. Gool: SURF: Speeded up robust features, Computer Vision and Image Understanding, 110(3), 346-359, 2008.

[9] D. Arthur and S. Vassilvitskii: $k$-means++: The advantages of careful seeding, Proceedings of Annual ACM-SIAM Symposium on Discrete Algorithms, 1027-1035, 2007.

[10] S. Lazebnik, C. Schmid, and J. Ponce, Beyond bags of features: Spatial pyramid matching for recognizing natural scene categories, Proceedings of IEEE Conference on Computer Vision and Pattern Recognition, 2169-2178, 2006.

[11] M. J. Swain and D. H. Ballard: Color indexing, International Journal of Computer Vision, 7(1), 11-32, 1991.

(2016年 8 月 19 日 受付)

(2017 年 1 月 6 日 採録) 
[Contact Address]

1-5-1 Chofugaoka, Chofu, Tokyo 182-8585, Japan

Department of Informatics, Graduate School of Informatics and Engineering, The University of Electro-Communications

TEL: +81-42-443-5280

E-mail: kazushi@uec.ac.jp

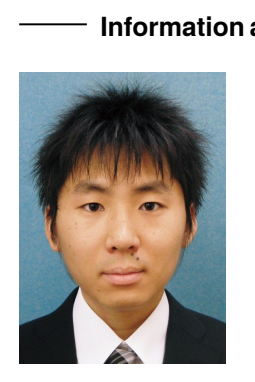

Kazushi OKAMOTO [member]

Kazushi Okamoto received the B.E. and M.E. degrees from Kochi University of Technology, Japan, in 2006 and 2008, respectively. He received the Ph.D. degree from Tokyo Institute of Technology, Japan, in 2011. From 2011 to 2015, he was an assistant professor with Chiba University, Japan. He is currently an assistant professor with The University of ElectroCommunications, Japan. His research interests include machine learning, data mining, and recommender system.

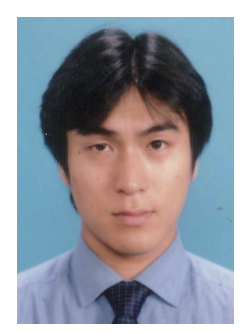

\section{Kazuhiko KAWAMOTO [member]}

Kazuhiko Kawamoto received the B.E., M.E., and D.E. degrees from Chiba University, Japan, in 1997, 1999, 2002, respectively. From 2002 to 2005 , he was an assistant professor with Tokyo Institute of Technology, Japan. From 2005 to 2009 , he was an associate professor with Kyushu Institute of Technology, Japan. He is currently an associate professor with Chiba University, Japan. His research interests include computer vision, pattern recognition, and statistical signal processing. 